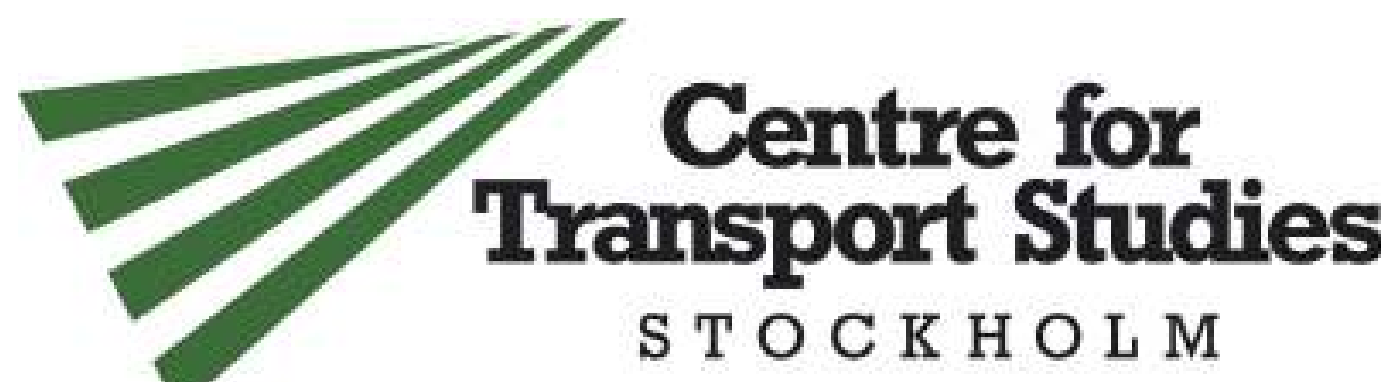

\title{
Mesoscopic Simulation for Transit Operations
}

\author{
Tomer Toledo, Oded Cats \\ Technion - Israel Institute of Technology, Haifa, Israel \\ Wilco Burghout, Haris N. Koutsopoulos \\ Royal Institute of Technology (KTH), Stockholm, Sweden
}

CTS Working Paper 2013:29

\section{Abstract}

This paper presents a transit simulation model designed to support evaluation of operations, planning and control, especially in the context of Advanced Public Transportation Systems (APTS). Examples of potential applications include frequency determination, evaluation of real-time control strategies for schedule maintenance and assessing the effects of vehicle scheduling on the level of service. Unlike most previous efforts in this area, the simulation model is built on a platform of a mesoscopic traffic simulation model, which allows modeling of the operation dynamics of large-scale transit systems taking into account the stochasticity due to interactions with road traffic. The capabilities of Mezzo as an evaluation tool of transit operations are demonstrated with an application to a realworld high-demand bus line in the Tel Aviv metropolitan area under various scenarios. The headway distributions at two stops are compared with field observations and show good consistency between simulated and observed data.

Keywords: Simulation; Public transport; Operations; ITS

Centre for Transport Studies 



\title{
Mesoscopic Simulation for Transit Operations
}

\author{
Tomer Toledo, Oded Cats
}

Technion - Israel Institute of Technology, Haifa, Israel

Wilco Burghout, Haris N. Koutsopoulos

Royal Institute of Technology (KTH), Stockholm, Sweden

\begin{abstract}
This paper presents a transit simulation model designed to support evaluation of operations, planning and control, especially in the context of Advanced Public Transportation Systems (APTS). Examples of potential applications include frequency determination, evaluation of real-time control strategies for schedule maintenance and assessing the effects of vehicle scheduling on the level of service. Unlike most previous efforts in this area, the simulation model is built on a platform of a mesoscopic traffic simulation model, which allows modeling of the operation dynamics of large-scale transit systems taking into account the stochasticity due to interactions with road traffic. The capabilities of Mezzo as an evaluation tool of transit operations are demonstrated with an application to a real-world high-demand bus line in the Tel Aviv metropolitan area under various scenarios. The headway distributions at two stops are compared with field observations and show good consistency between simulated and observed data.
\end{abstract}




\section{INTRODUCTION}

Public transportation systems are increasingly complex, incorporating diverse travel modes and services. As a result, various Advanced Public Transportation Systems (APTS), designed to assist operators, have been developed and implemented (Casey et al. 2000). The need to integrate and efficiently operate these systems poses a challenge to planners and operators. As new technologies and applications are proposed, tools to assist in their development and evaluation prior to field implementation are needed.

In the context of general traffic operations, simulation models have been established as the primary tool for evaluation at the operational level. Most of the advances in these models related to transit systems have focused on implementation of transit signal priority (Khasnabis et al. 1996, Liu et al. 1999, Chang et al. 2003, Werf 2004, Lee et al. 2005, Fernandez et al. 2007), operation of bus stops (Liu et al. 1999, Silva 2001, Ding et al. 2001, Werf 2004, Fernandez et al. 2007) and bus lanes (Liu et al. 2006, Fernandez et al. 2007).

Transit simulations provide a dynamic perspective on transit operations, enabling comparisons of various scenarios and representation of complex interactions between the network components: general traffic, transit vehicles and passengers. However, although simulation models can have many advantages for public transportation research, there has not been much effort in the development of transit simulation models. Algers et al. (1997) reviewed 32 microscopic traffic simulation models and reported that only 52\% represent public transportation at all, $42 \%$ model transit priority, and only $6 \%$ model transit traveler information systems. At the same time, the majority of users they interviewed were interested in large-scale applications at the urban or regional context, and that these users ranked 
modeling of public transportation the second most important capability in traffic simulation models. In the time since their study was conducted, several microscopic traffic simulation models have significantly enhanced their transit capabilities. Boxill and $\mathrm{Yu}(2000)$ report that the capability of existing simulation models to effectively simulate APTS applications in large networks is limited. While they found that few microscopic models simulate well the local impacts of APTS, none of the mesoscopic models they reviewed had any transit simulation component at all.

As noted above, most efforts in modeling public transportation and APTS have focused on microscopic simulations. However, these models are inefficient when applied to large-scale applications because of the unnecessary level of detail and extensive computational effort they require. In contrast, mesoscopic simulation models, which represent individual vehicles but avoid detailed modeling of their second-by-second movement, may be useful for systemwide evaluation of transit operations and APTS, as they are for general traffic.

This paper reports on the development of a mesoscopic transit simulation model designed to support evaluation of operations planning and control, especially in the context of APTS. Examples of potential applications include frequency determination, evaluation of real time control strategies for schedule maintenance, restoration from major disruptions and assessing the effects of vehicle scheduling on the level of service. The rest of the paper is organized as follows: First, Mezzo, the mesoscopic traffic simulator that is used as a platform for the development of the transit simulator is described. Next, the overall framework and implementation details of the transit simulation model are presented. The application of the transit simulator is demonstrated with an application to a high-demand bus line in the TelAviv metropolitan area. The demonstration includes a validation, study of travel time 
variability and demand levels and a sensitivity analysis showing the impact of the recovery time policy on performance. Finally, a discussion and concluding remarks are presented.

\section{MEZZO}

The transit simulation model is built within the platform of Mezzo, a mesoscopic traffic simulation model (Burghout 2004, Burghout et al. 2006). Mezzo is an event-based simulator, which models vehicles individually, but does not represent lanes explicitly. Links in Mezzo are divided into two parts: a running part, which contains vehicles that are not delayed by the downstream capacity limit; and a queuing part, which extends upstream from the end of the link when capacity is exceeded. The boundaries between the running and queuing parts are dynamic and depend on the extent of the queue. Vehicles enter the exit queue in the order that they complete their travel in the running part. The earliest exit time is calculated as a function of the density in the running part only.

Travel times on the running part are determined by a speed-density function. The default function is:

$V(k)=\left\{\begin{array}{cc}V_{\text {free }} & k<k_{\text {min }} \\ V_{\text {min }}+\left(V_{\text {free }}-V_{\text {min }}\right) \cdot\left[1-\left(\frac{k-k_{\min }}{k_{\text {max }}-k_{\text {min }}}\right)^{a}\right]^{b} & k \in\left[k_{\min }, k_{\max }\right] \\ V_{\text {min }} & k>k_{\text {max }}\end{array}\right.$

Where $V_{\text {free }}$ and $V_{\min }$ are the free flow speed and minimum speed, respectively. $k$ is the density in the running part of the link. $k_{\min }$ and $k_{\max }$ are the minimum and maximum densities 
thresholds. $a$ and $b$ are parameters. This speed-density function determines that the vehicle moves at free flow speed when the density is lower than $k_{\min }$ and has a constant minimum speed if the density exceeds $k_{\text {max }}$.

At the downstream part of the link, the vehicles join a single queue of vehicles waiting to move out of the link. Queue servers process the vehicles in this queue and pass them on to the next link if it is not full. Separate queue servers with their corresponding capacities are used for each turning movement in order to capture link connectivity and lane channeling. Each turning movement server searches backwards from the head of the queue for vehicles that intend to use the turn movement it regulates and processes them in sequence. A maximum queue look-back limit may be defined for each turning movement in order to represent dependencies among turning movements (e.g. when a queue in one movement blocks access to the lanes used by another turn movement). Turning servers are modeled stochastically with truncated normal service times.

Vehicles in Mezzo are generated at mean rates specified by time-dependent OriginDestination (OD) flow matrices. Pre-trip route choices follow the multinomial logit model with a set of pre-defined routes and historical link travel times. En-route, drivers may switch their routes in response to information they receive. The route switching model also uses the multinomial logit model structure. Information received by the drivers is used to update the travel times and routes sets that drivers use in evaluating the utilities of the various routes. 
Mezzo is implemented modularly using the object oriented programming (OOP) approach in order to enable further enhancements and developments. Each entity in the simulation model (e.g. node, queue, vehicle, OD pair) is represented as an object with its related variables and functions. As an event-based simulation model, the event list calls the Action objects at the appropriate times at which they are booked. A complete description of the structure of Mezzo and its implementation details is presented in Burghout (2004). Mezzo is also capable of hybrid microscopic-mesoscopic simulation (Burghout et al. 2005) and has been applied in evaluating bus-priority in adaptive signal control (Burghout and Wahlstedt 2007).

\section{TRANSIT SIMULATION}

\subsection{Object framework}

Mezzo was extended to simulate transit operations, based on the framework shown in Figure 1, which presents only the six transit-oriented classes: Bus Type, Bus Vehicle, Bus Line, Bus Route, Bus Trip and Bus Stop. The Bus Type objects define the characteristics of the different types of vehicles, such as length, number of seats and passenger capacity. Each Bus Vehicle object inherits the attributes of the specific bus type and general attributes and functions that are relevant for each vehicle in the simulation. In addition, bus vehicles maintain a list of their scheduled trips, which allows explicit modeling of trip chaining including layover and recovery times in the trip sequence. During the simulation, the Bus Vehicle object maintains updated passenger loads and determines crowding levels and the maximum number of passengers that may board at each stop.

A bus line is defined by its origin and destination terminals and the sequence of stops that it serves in between. The Bus Line object holds information on scheduled departure times from 
the origin and keeps track of the list of active trips as it may have several simultaneously. Each bus line indicates the vehicle type that should be assigned for this service. It may also store a subset of the stops that serve as possible time point stops and the appropriate holding strategy. The unique route in terms of a sequence of links travelled is stored by a Bus Route object, which is a sub-class of the general Route object.

The bus line service is performed through individual bus trips. A Bus Trip object has a unique bus line, while the number of bus trips per bus line is determined by the number of vehicles that depart. The Bus Trip object maintains the schedule of expected arrival times at each stop for the specific trip. During the simulation, it calculates the actual departure time from the origin terminal and records arrival times at bus stops.

The Bus Stop objects hold the information on each bus stop in the network. The bus stop is identified by the link it is located on and its position on that link. It also contains information on physical characteristics, such as the length and type (in-lane or bay stop), and holds a list of bus lines that serve this stop. During the simulation it monitors time of the last arrival to the stop for each bus line in order to enable calculation of the actual headways between sequential buses. These headways are in turn used to calculate passenger arrivals, the number of waiting passengers per line and their waiting times. Every time a bus arrives, the Bus Stop object calculates the dwell time for the bus and the number of waiting passengers that were not able to board the bus. The Bus Stop and Link objects have a one-to-many relationship: A bus stop is associated with a specific link, while each link may have several stops on it. 


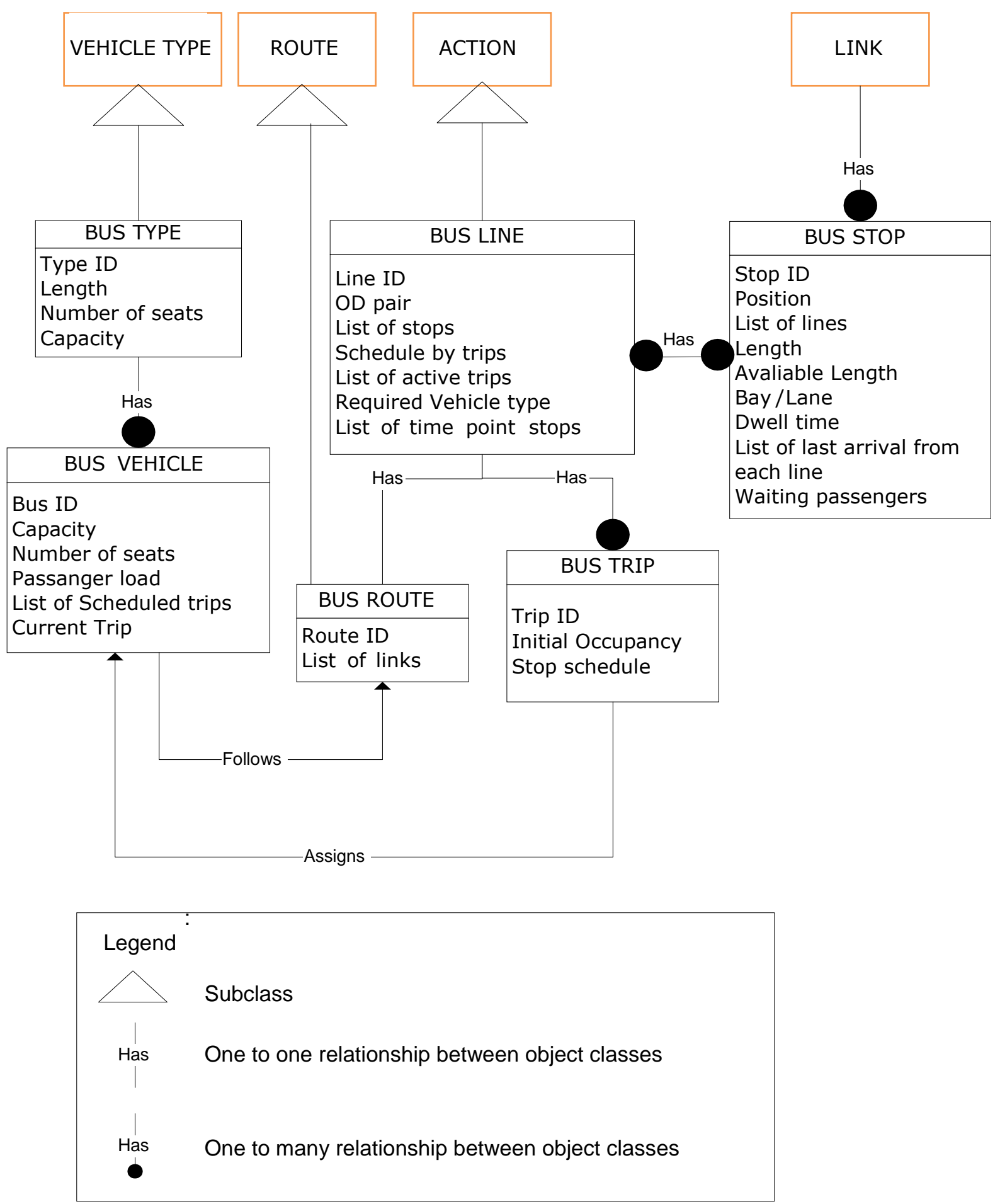

Figure 1: Object-oriented framework for the transit simulation structure 


\subsection{Simulation flow}

As mentioned above, Mezzo is an event-based simulation model. As such, the time clock of the simulation progress from one event to the next according to a chronological list of events that refers to the relevant objects. At the start of the simulation, all objects are initialized and some of them register an event. The execution of most events trigger the generation of new subsequent events. The transit simulation introduces several new event types. Figure 2 shows a flowchart of the transit simulation process. On initialization of the simulation run, a list of the bus lines that are modeled is read and the corresponding Bus Line, Bus Route and Bus Type objects are created. At this stage, events are registered in the event list for the next scheduled departure for each line. When a scheduled trip departure event is activated the Bus Trip object is generated. A bus vehicle is assigned to this trip. If the assigned vehicle is not yet in service (in case that this trip is the first on its trip chain) then a Bus Vehicle object is generated and assigned the properties of the required bus type. It then enters the first link on its route. This is also the case if the Bus Vehicle object already exists and is available to depart. In case that the bus vehicle is not yet available to depart (i.e. has not completed the recovery time from its previous trip), the trip departure is deferred until the vehicle becomes available. 


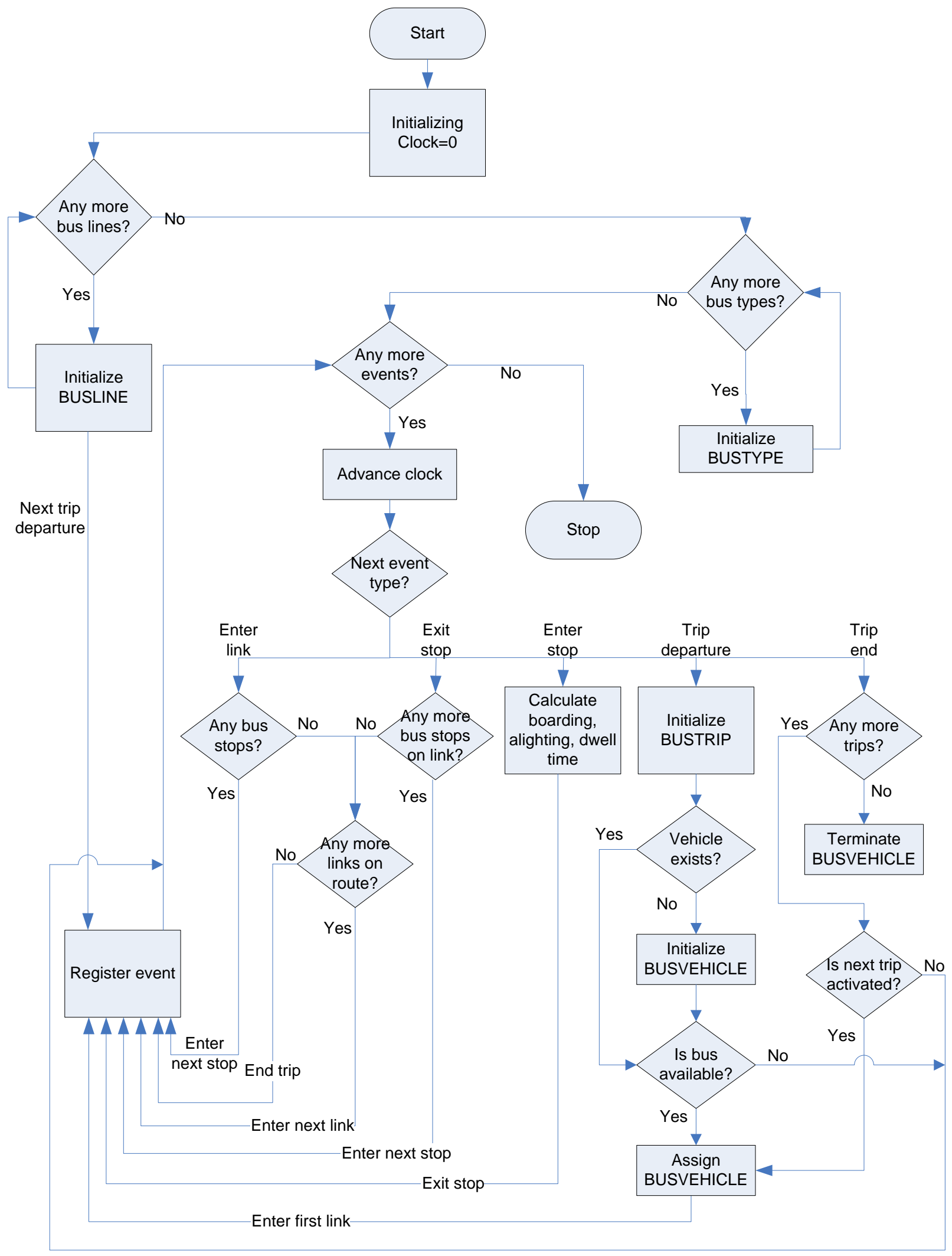

Figure 2: Flowchart of the transit simulation process 
A bus vehicle that enters a link on its route checks whether or not there are bus stops to be serviced on this link. If there are no stops on the link, the link exit time is calculated and an event to enter the next link is added to the event list. Link travel times are calculated based on traffic conditions as for all vehicles in Mezzo. If there is a stop on the link, the travel time to the stop is calculated and an event to enter the stop is generated with the appropriate arrival time. The driving time to the stop is a calculated as a proportion of the link travel time, depending on the location of the stop. Once the bus enters a stop, the dwell time is calculated. Based on the dwell time and taking into account any control strategies that may be implemented the timing for a new event to exit the bus stop is determined. When the bus exits the stop, similarly to the event of entering a link, Mezzo checks if there are any more stops on the link and calculates the driving time to the next stop or to the end of the link based on the current traffic conditions and on the distance to the next stop or the end of the link. An event to enter the next stop or to exit the link is generated.

Finally, when the bus arrives at the end of its route and the trip ends, Mezzo checks whether or not there is an additional trip for this bus vehicle. If so, and the next trip has already been activated (i.e. the trip scheduled departure time has already passed), the bus vehicle is assigned to the next trip and enters its first link. In the case that the next trip is not activated the bus vehicle waits until the scheduled departure time. The bus vehicle is deleted if there are no more trips on the vehicle scheduling of this vehicle.

The main simulation loop is designed to support the implementation of control strategies, which requires additional steps. Each object that is a potential subject for control strategy is indicated by a flag. Every time that an event is executed, the model checks whether a control 
strategy is defined for this type of event, and if so, executing the control logic to determine the appropriate action. For example, if holding control is in place, for every bus that enters a stop, the simulation checks whether the bus stop is listed as a time point stop for the specific trip and if it is, for how long the bus should be held (if at all). The result of this check would affect the time associated to the stop exit event that will be generated.

Outputs from the simulation include stop level statistics, such as early and late arrivals, dwell times, numbers of boarding and alighting, bus loads and travel times between stops. Aggregations at the level of the trip, the vehicle or the line, such as schedule adherence, headway and passenger wait time distributions, load profiles and other level of service measures are also computed.

\subsection{Implemented models}

The additional transit simulation components were designed to include detailed representation of the operations of public transportation including its basic attributes such as travel times, dwell times, boarding and alighting processes and recovery times. The assumptions made about these processes are critically important because they dictate the demand and supply representation and also the resulting level of service measures, such as passenger waiting times (Bowman and Turnquist 1981). This section describes the main components of the transit simulation model: the passenger arrival and alighting processes, dwell time functions and trip chaining. 


\subsubsection{Passenger arrival and alighting processes}

Passenger demand is represented by two components: the arrival rates at stops of passengers for each line and the demand to get off the bus at each stop. This level of representation is detailed enough to support study of the impacts of demand on service times and on crowding levels, while relying on aggregate modeling of transit users, avoiding explicit generation of individual passengers.

Thus, the inputs to the model are time-dependent matrices of passenger arrival rates and of alighting fractions for each bus stop and each bus line. They are used as mean values in stochastic arrival and alighting processes. We adopt the approach in most studies of these processes that assume that passenger arrivals follow the Poisson distribution (Fu and Yung 2002, Dessouky et al. 2003):

$B_{i j k} \sim \operatorname{Poisson}\left(\lambda_{i j t_{k}}, h_{i j k}\right)$

Where $B_{i j k}$ is the number of passengers wishing to board line $i$ at stop $j$ on trip $k . \lambda_{i j t_{k}}$ is the arrival rate for line $i$ at stop $j$ during the relevant time period $t_{k} . h_{i j k}$ is the time headway on line $i$ at stop $j$ between the preceding bus (on trip $k-1$ ) and the bus on trip $k$. In case that the passenger arrival process takes place over periods with different arrival rates (i.e. the relevant headway is spread over two or more time periods) the number of passengers wishing to board is calculated as the sum of the generations in these time periods. 
The passenger alighting process is assumed to follow a Binomial distribution (Morgan 2002, Liu and Wirasinghe 2001):

$A_{i j k} \sim \operatorname{Binomial}\left(L_{i j k}, P_{i j t_{k}}\right)$

$A_{i j k}$ is the number of alighting passengers from line $i$ at stop $j$ on trip $k . \alpha_{2} L_{i j k}$ is the load on arrival at stop $j$ on the bus on trip $k$ of line $i . P_{i j t_{k}}$ is the probability, during the relevant time period $t_{k}$, that a passenger on line $i$ will get off the bus at stop $j$.

\subsubsection{Dwell times}

Trip travel times consist of two parts: running times and dwell times. Dwell times include the time needed for the doors to open, boarding and alighting of passengers, the closing of the doors and the bus to get off the stop. The default dwell time function implemented in the model is based on the one adopted in the Transit Capacity and Quality of Service Manual (TCRP 2003). With this function, the time needed for passengers to board and alight is calculated separately for each door. The overall dwell time is determined by the door that has the longest service time (Lin and Wilson 1992). In addition, the function differentiates between stops that are placed in-lane and those that use a bus bay, which require longer dwell times due to the time needed for the bus to re-join traffic on the regular lane when exiting the stop. The model also assumes that dwell times increase when buses alight and board passengers outside of the physical stop (e.g. because the stop is occupied by other buses). For standard buses, the resulting dwell time function is given by: 
$D T_{i j k}=\beta_{1}+\max \left(P T_{i j k}^{f r o n t}, P T_{i j k}^{r e a r}\right)+\beta_{2} \cdot \delta_{j}^{b a y}+\beta_{3} \cdot \delta_{i j k}^{f u l l}+v_{i j k}$

Where, $D T_{i j k}$ is the dwell time for line $i$ at stop $j$ on trip $k . P T_{i j k}^{d}$ is the total passenger service time on door $d \in\{$ front, rear $\}$, which depends on the numbers of boarding and alighting passengers and the crowding level on the bus. $\delta_{j}^{\text {bay }}$ is a bay stop indicator which takes the value 1 if the bus stop is in a bay and 0 otherwise. $\delta_{i j k}^{\text {full }}$ is an indicator for the available physical space at the stop, which takes the value 1 if all the stop is completely occupied and 0 otherwise. $\beta_{1}, \beta_{2}, \beta_{3}$ are parameters and $v_{i j k}$ is an error term.

The passenger service time is the main component of the dwell time function. In the case that boarding is allowed only on the front door and alighting is possible from both doors the following functions are used:

$$
\begin{aligned}
& P T_{i j k}^{\text {front }}=\alpha_{1} \cdot p_{\text {front }} \cdot A_{i j k}+\alpha_{2} \cdot B_{i j k}+\alpha_{3} \cdot \delta_{i j k}^{\text {crowded }} \cdot B_{i j k} \\
& P T_{i j k}^{\text {rear }}=\alpha_{4} \cdot\left(1-p_{\text {front }}\right) \cdot A_{i j k}
\end{aligned}
$$

Where $p_{\text {front }}$ is the fraction of passengers that alight from the front door. $\alpha_{1}, \alpha_{2}$ and $\alpha_{3}$ are parameters. $\delta_{i j k}^{\text {crowded }}$ is a crowding indicator, which takes the value 1 if the number of passengers on the bus exceeds the number of seats, and 0 otherwise. 
In addition, the model supports the implementation of control strategies at stops. In the presence of control strategies, the departure time from the stop is given by:

$$
E T_{i j k}=\max \left(A T_{i j k}+D T_{i j k}, C T_{i j k}\right)
$$

Where $E T_{i j k}, A T_{i j k}$ and $C T_{i j k}$ are the departure time, actual arrival time and the time resulting from the control strategy implemented for line $i$ on trip $k$ from stop $j$, respectively.

\subsubsection{Trip chaining}

Vehicles in Mezzo are assigned an origin and a destination and follow a route between them. They are normally terminated when they reach their destination. In contrast, transit vehicles are not terminated after each trip, but follow a schedule that includes a sequence of trips. The ability to model the chain of trip the vehicle undertakes allows the simulation to model the accumulated impact of the planned schedule on the level of service. Thus, the actual departure time of a chained trip is calculated as the later between the scheduled departure time and the time the bus vehicle is available to depart after it completed its previous trip and some recovery time:

$$
D P T_{b k}=\max \left(S T_{b k}, A T_{b, k-1}+R T_{\min }+\varepsilon_{b k}\right)
$$

Where $D P T_{b k}$ and $S T_{b k}$ are the actual and scheduled departure times for trip $k$ by bus vehicle $b$, respectively. $A T_{b, k-1}$ is the arrival time of bus $b$ from the previous trip at the origin terminal of the current trip. $R T_{\min }$ is the minimum recovery time required between trips. $\varepsilon_{b k}$ 
is a lognormally distributed error term representing the stochastic part of the recovery time, capturing factors such as possible delay for the first trip of the vehicle as it comes from the garage or depot or effects of departure supervision for the intermediate trip chain.

\section{CASE STUDY}

\subsection{Bus line description}

In order to demonstrate its capabilities, the transit simulator is applied to a case study to evaluate the operations of line 51 in the Tel Aviv metropolitan area in Israel. The line route and demand profiles for the inbound and outbound directions are shown in Figure 3 and Figure 4, respectively. This high demand urban line connects a dense satellite residential city to the CBD. Its 14 kilometer long route follows a heavily congested urban arterial. The line includes 30 stops in the inbound direction and 33 in the outbound direction. The scheduled headway during the peak period is 8 minutes and the average running time is 49 minutes inbound and 41 minutes outbound. 


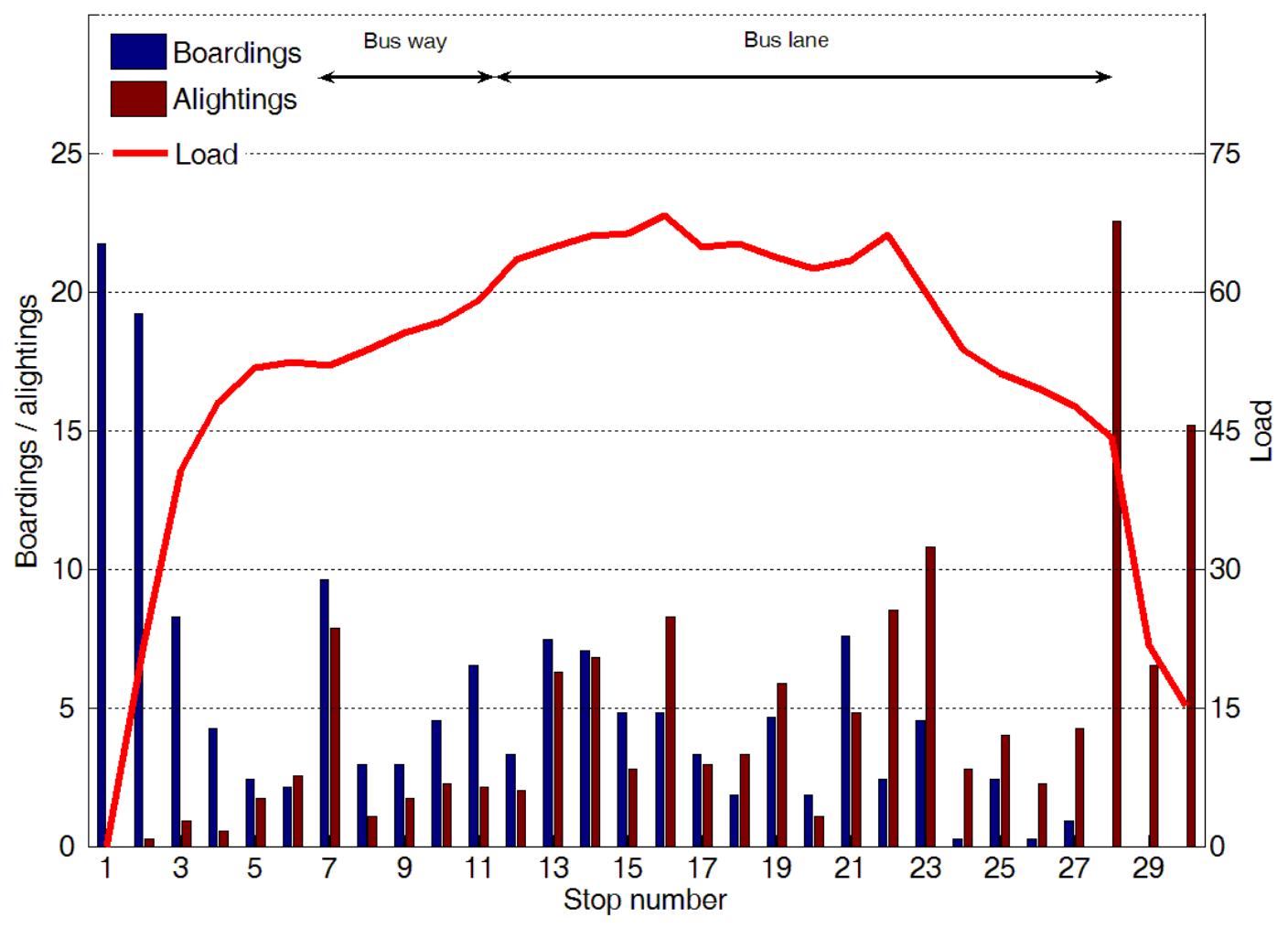

Figure 3: Schematic route and load profile for inbound line 51 


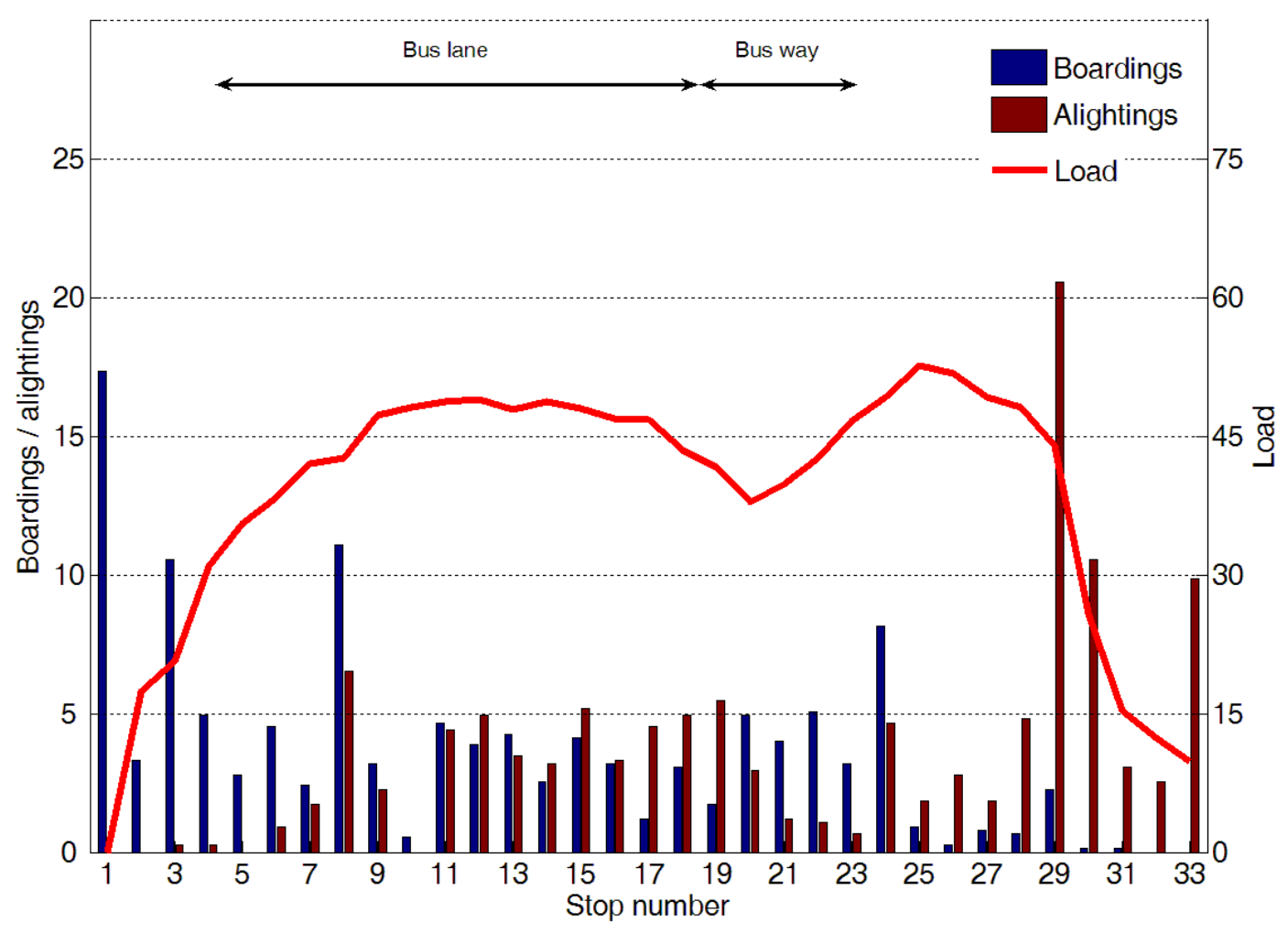

Figure 4: Schematic route and load profile for outbound line 51

\subsection{Validation results}

The outputs of the simulation were tested against real-world data. Video traffic records were available from two bus stops - Stop 28 stop on the inbound direction and stop 4 on the outbound direction for the period 06:30-08:30. Figure 5 shows the observed and simulated headway distributions for these two stops, figure 6 shows the cumulative distribution functions of headways. , 

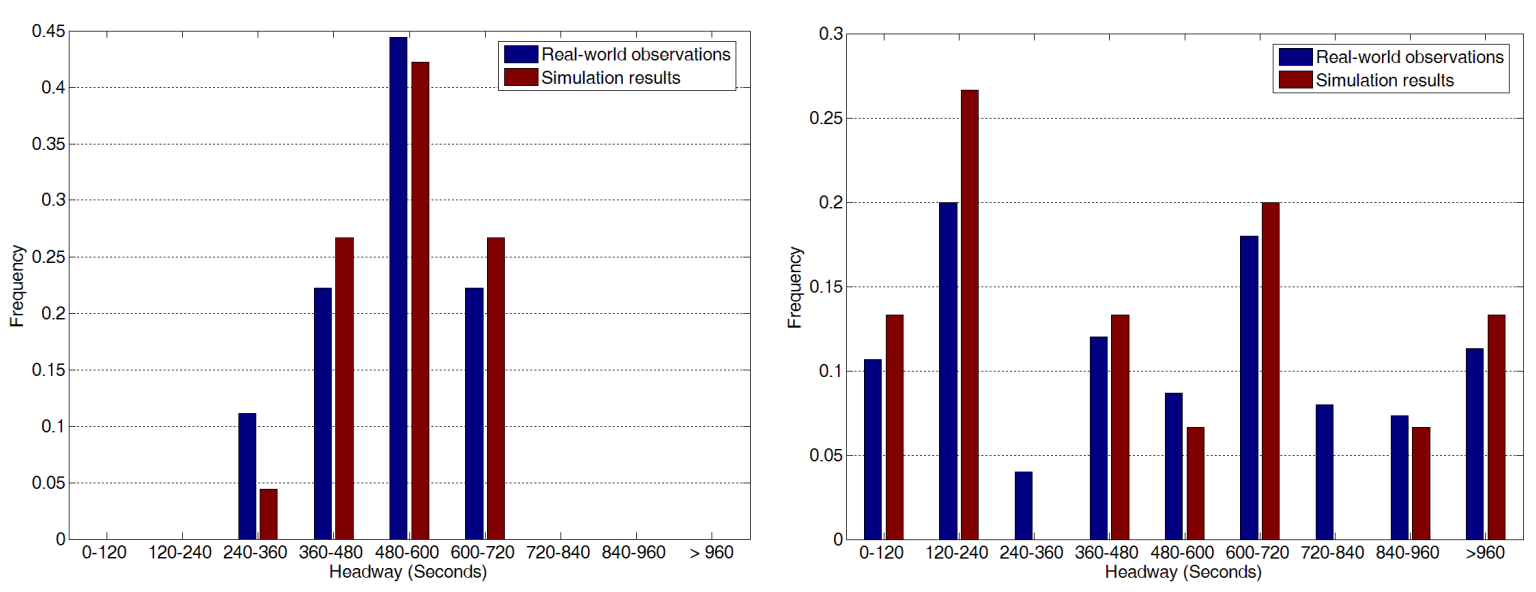

Figure 5: Headway distributions at stops 4(left) and 28 (right)
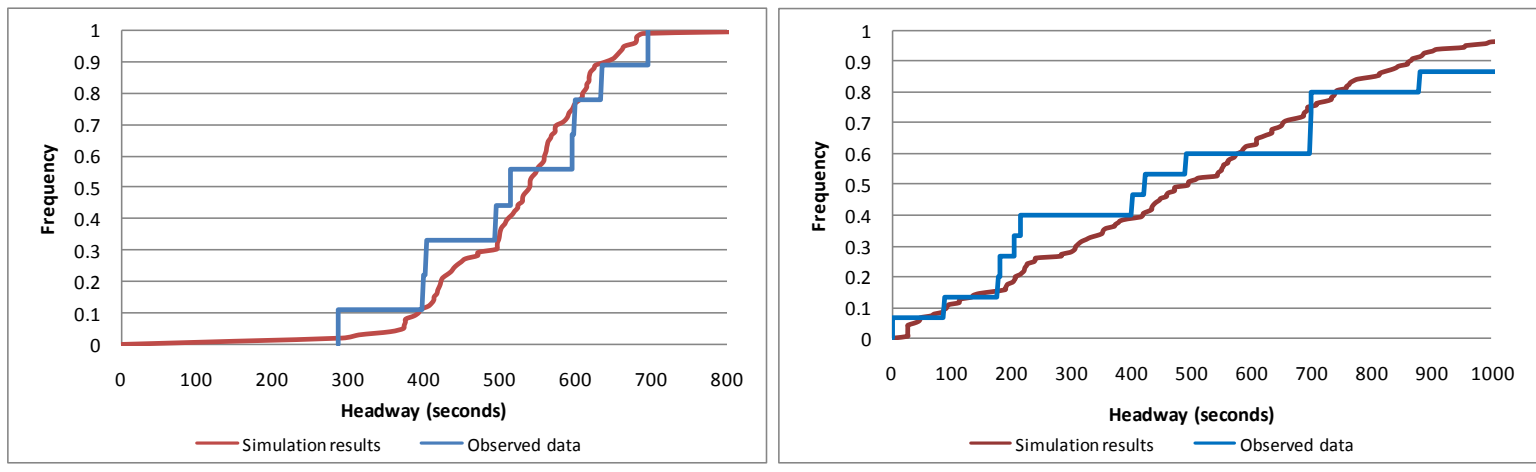

Figure 6: CDF of headways at stops 4(left) and 28(right)

Two-sample Kolmogorov-Smirnov tests were conducted in order to compare the distributions of the observed and simulated headways at these two stops. The results are presented in table 1. The test results are for both stop 4 and 28 , that at the 0.05 level, $\mathrm{D}<\mathrm{D}_{0.05}$ so the null hypothesis that the observed and simulated headways are derived from the same distribution, cannot be rejected. 
Table 1: Kolmogorov-Smirnov statistics for stop 4 and 28

\begin{tabular}{c|ccc} 
& $\mathbf{n}$ & $\mathbf{D}_{\mathbf{0 . 0 5}}$ & $\mathbf{D}$ \\
\hline Stop 4 & 15 & 0.338 & 0.212 \\
Stop 28 & 8 & 0.457 & 0.121
\end{tabular}

In addition, the coefficients of variation for observed and simulation data were compared. For stop 28 the coefficient of variation is 0.80 for the observed data and 0.60 for the simulated data, whereas for stop 4 they are 0.26 and 0.20 , respecitvely. As can be expected, the coefficient of variation of the headway distribution is higher at stop 28 than for stop 4, which is much closer to the origin terminal. This result suggests that implementation of control strategies, such as headway or schedule-based holding of buses at time points may be useful for this line. The results suggest a slight underestimation of the headway variability, which may be the result of the implicit representation of the impact of background car traffic on link travel time variability. Therefore, car traffic impacts do not propogate over links, ignoring the correlation of travel time variabilities for consecutive links. In the future, background traffic will be modeled explicitly.

A comprehensive validation of the model requires many more observations and for a larger number of stops along the line. However the presented evaluation shows that the simulation results are consistent with field observations. 


\subsection{Experiment}

The demonstration experiment included study of the impact of two factors on the line performance: the passenger demand and travel time variability. Table 2 summarizes the values of these factors. Travel time variability values are based on those found in literature (Taylor 1982, Fu and Yang 2002, Dessouky et al. 2003, Ceder 2007). Nine different scenarios were simulated, one for each possible combination of the factors. For each scenario 10 fourhour simulation runs were conducted for a four hour period between 6AM and 10AM. The execution time for a single run was about 45 seconds, and so the 90 runs took about 67 minutes to complete. All of the simulation runs were executed on Pentium 4 with $3.01 \mathrm{GHz}$ and $512 \mathrm{MB}$ ram under windows. The reported results are the average of the 10 replications for each scenario.

Table 2: Factors and their levels in the experiment

\begin{tabular}{|l|l|}
\hline \multicolumn{1}{|c|}{ Factor } & Levels \\
\hline Passenger demand & $80 \%, 100 \%, 120 \%$ of observed demand profile \\
\hline Travel time variability & $80 \%, 100 \%, 120 \%$ of mean travel time \\
\hline
\end{tabular}

In the case study running times between stops were assumed to follow lognormal distributions, with means equal to the scheduled times. At both trip ends, recovery times were calculated based on the $85^{\text {th }}$ percentile of the trip travel times, calculated according to the lognormal distribution. These recovery times were then used as minimum requirements in determining the trip assignment for each bus vehicle, while the layover times are already 
integrated into the scheduled times. In addition, a sensitivity analysis on the layover policy was conducted. The trip chain was designed with two additional recovery policies: using the $55^{\text {th }}$ and the $70^{\text {th }}$ percentile of total travel times. These policies were implemented with the intermediate demand and variability levels.

\subsection{Results}

The detailed representation of the bus operations in the simulation allows evaluation of its performance ranging from the level of a single run to the overall system performance. At the most detailed level, Figure 7 presents a time-space diagram showing the trajectories of two selected buses (buses 12 and 13 out of the 16 assigned bus vehicles) in service on line 51 during the study period. The continuous lines are the simulated trajectories compared with the scheduled trajectories displayed by the broken lines. In the figure, both buses make three trips. Bus 12 is ahead of schedule on its first trip, is increasingly late on the second and on time on the third. The well-known bunching phenomenon (e.g. Abkowitz and Tozzi 1987) is reproduced by the simulation as is evident in the second and third trips, when buses 12 and 13 arrive simultaneously as they progress along their route. Recovery times between trips at both terminals are also apparent in the figure, as both buses conducted three sequential trips. 


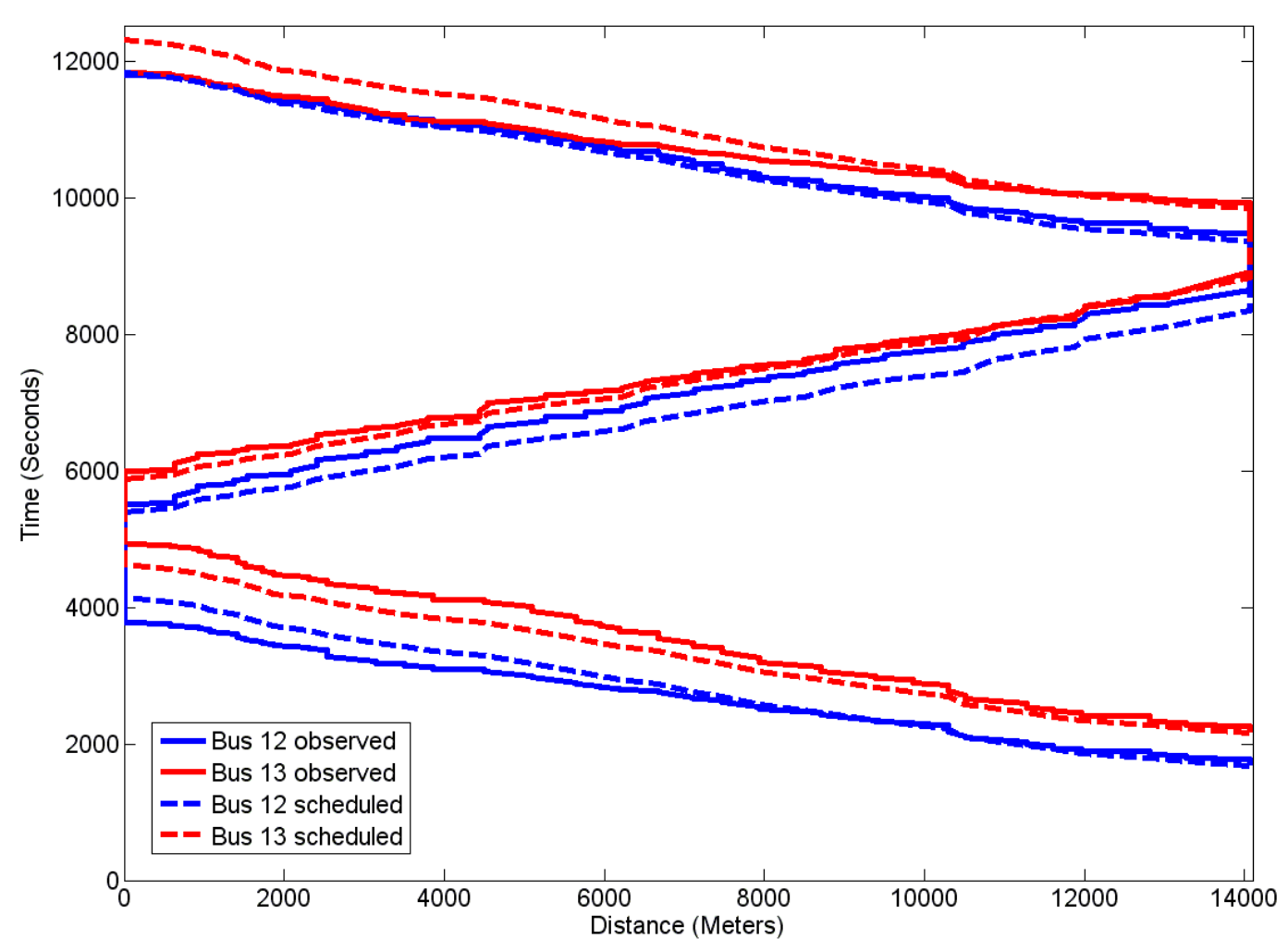

Figure 7: Time-space diagram of buses on service in line 51

A phenomenon in transit systems that may have significant impact on levels of service is the accumulation of variability in travel times as buses progress through their schedules. Figure 8 demonstrates the evolution of headway variability at the various stops along the inbound route. As the standard deviation of the headway increases along the route, the on-time performance statistic decreases - It drops from $100 \%$ to $48 \%$. Following Ceder (2007), a bus is considered to adhere to schedule at a specific stop, if it arrives between one minute early and four minutes late compared to its scheduled arrival. 


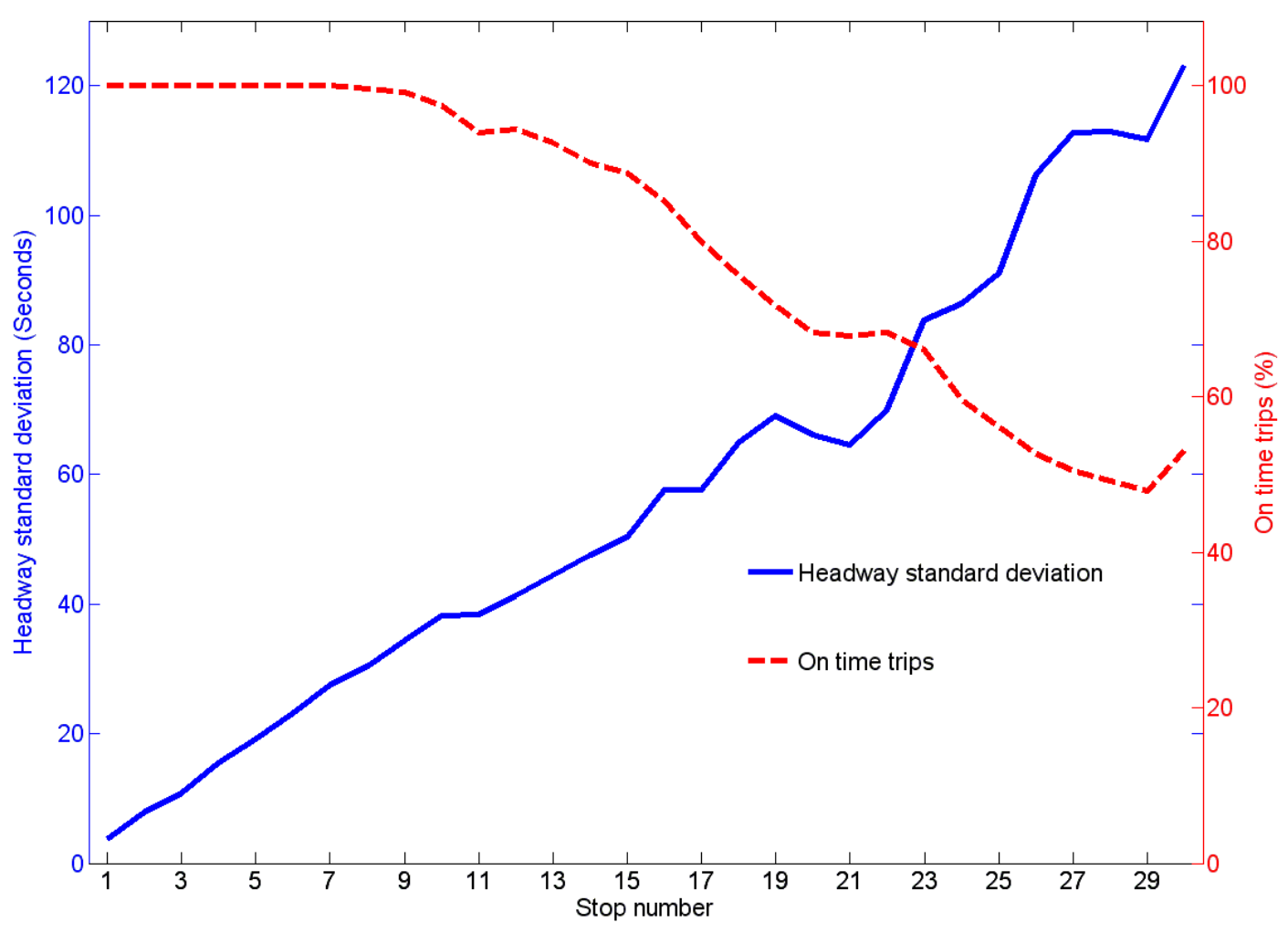

Figure 8: Standard deviation of the headway and on-time performance along the inbound route

Figure 9 shows an example of the load profiles of the outbound route for two successive buses. The leading bus had a long headway followed by a bus with a short headway. For comparison the expected load profile for the planned headway, which was tested by a simulation run with deterministic conditions (constant running times and dwell times) is presented as well. It can be seen that the actual load profile varied significantly from the one expected under deterministic conditions: the first bus with high headway had to pick up all the passengers that had accumulated, which resulted in longer dwell times and caused the following bus that had fewer passengers and therefore shorter dwell times to catch up with it. This trend was restrained in the intermediate stops, as the first bus with the long headway 
reached its capacity (70 passengers) and left waiting passengers behind. As a result, the second bus with the short headway had to pick up more passengers than expected according to its headway. Finally, the headway at the destination terminal was only two minutes, instead of 8 minutes, as planned. From the passenger point of view, being unable to board overcrowded buses are sources for unreliable and inconvenient service.

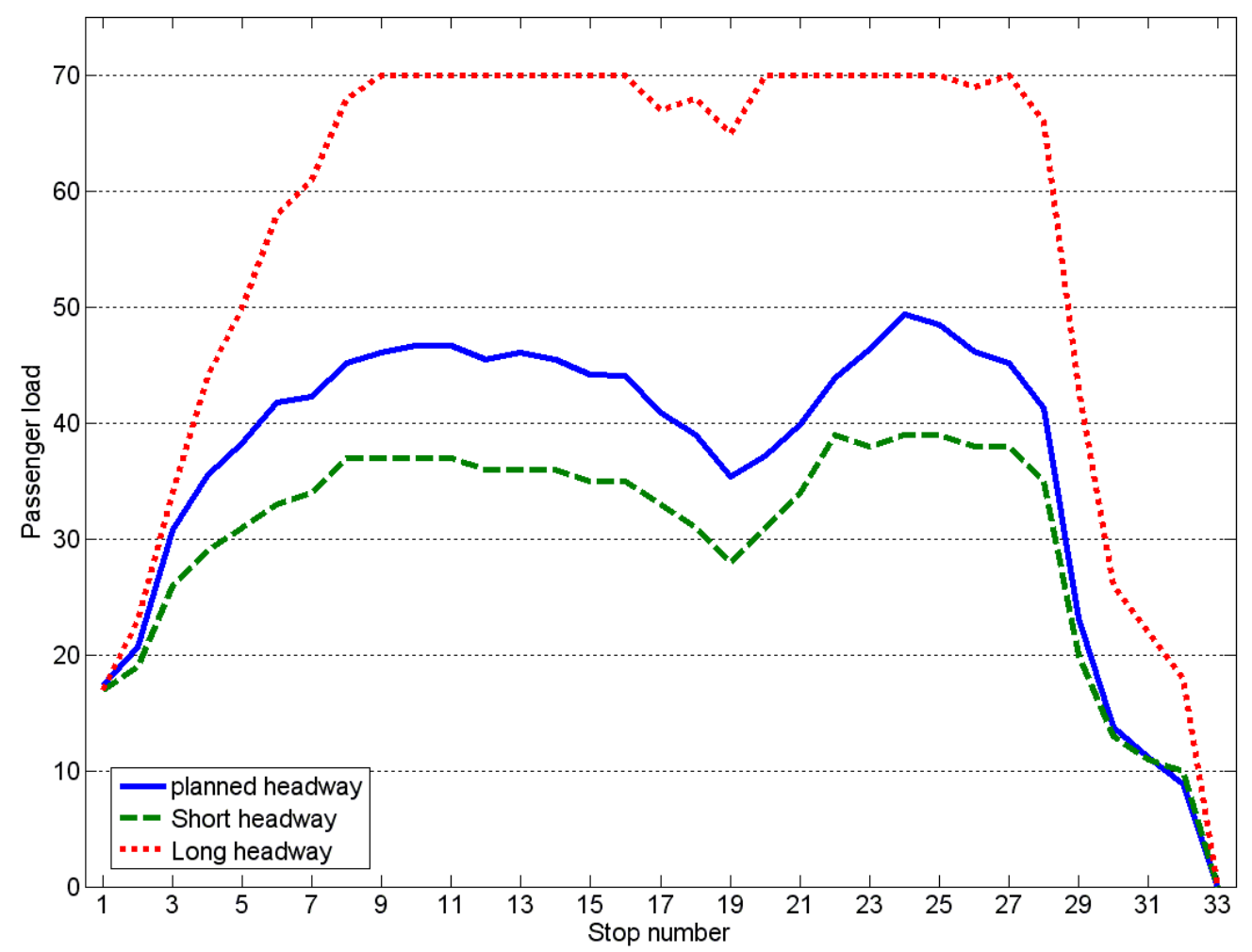

Figure 9: Planned and experienced load profiles for bunched buses (outbound route)

At the system level, several measures of performance were calculated for each scenario. Table 3 summarizes these measures for the various scenarios. The variability of headways is the main measure for evaluating transit reliability in particular for short-headway services, when 
bus bunching occurs. The headway variability was calculated for each stop along the route.

The reported statistics are the mean values across all stops in each direction.

Table 3: Service measures of performance under various scenarios

\begin{tabular}{|c|c|c|c|c|c|c|c|}
\hline \multicolumn{2}{|c|}{ Scenario } & \multicolumn{6}{|c|}{ Measure of performance } \\
\hline Demand & $\begin{array}{c}\text { Travel } \\
\text { time } \\
\text { variability }\end{array}$ & $\begin{array}{l}\text { Inbound } \\
\text { headway } \\
\text { standard } \\
\text { deviation } \\
\text { (seconds) }\end{array}$ & $\begin{array}{l}\text { Outbound } \\
\text { headway } \\
\text { standard } \\
\text { deviation } \\
\text { (seconds) }\end{array}$ & $\begin{array}{c}\text { Early } \\
\text { arrivals } \\
(\%)\end{array}$ & $\begin{array}{c}\text { Late } \\
\text { arrivals } \\
\qquad \%)\end{array}$ & $\begin{array}{l}\text { On-time } \\
\text { trips }(\%)\end{array}$ & $\begin{array}{l}\text { Passengers } \\
\text { unable to } \\
\text { board per } \\
\text { stop }\end{array}$ \\
\hline Low & Low & 63.6 & 69.7 & 20.1 & 22.1 & 57.6 & 0.00 \\
\hline Low & Moderate & 65.8 & 87.9 & 16.9 & 17.6 & 65.6 & 0.00 \\
\hline Low & High & 85.5 & 97.7 & 19.7 & 20.5 & 59.8 & 0.00 \\
\hline Moderate & Low & 49.5 & 58.8 & 10.0 & 17.2 & 72.7 & 0.19 \\
\hline Moderate & Moderate & 57.0 & 78.8 & 11.3 & 17.6 & 71.1 & 0.19 \\
\hline Moderate & High & 76.4 & 93.0 & 8.9 & 21.8 & 69.3 & 0.40 \\
\hline High & Low & 47.1 & 44.9 & 5.1 & 14.7 & 80.2 & 1.58 \\
\hline High & Moderate & 55.1 & 71.5 & 4.8 & 11.4 & 83.8 & 1.85 \\
\hline High & High & 68.7 & 80.5 & 5.0 & 16.2 & 78.9 & 1.29 \\
\hline
\end{tabular}

The headway variability increases with the level of variability of running times between stops.

It is evident that higher travel time variability level results in a less regular service, with less stop arrivals that adhere to the planned headway. Higher travel time variability causes higher frequency of extreme values, which represent bunching. Service regularity can be measured 
as the percentage of headways that are between $50 \%$ and $150 \%$ of the planned headway (Nakanishi, 1997). The service regularity was decreased from $77 \%$ under the low variability travel time to $72 \%$ under the high variability travel time.

In contrast, the headway variability did not increase with demand level. This result is perhaps counter-intuitive. It seems to derive from the high demand load. Initially, the headway variability increases with the demand level because of the connection between the mean dwell time and the dwell time variability implied in the Poisson passenger arrival process. However, at a certain level of demand, buses become too crowded to allow all waiting passengers to board, as the simulation model takes into account the restricted capacity. Therefore, the dwell time variability decreases. In order to examine this explanation, an additional demand scenario of half of the observed demand profile was run. Figure 10 presents the relation between headway variability and the demand level, which supports the above-mentioned hypothesis: the headway variability increases with the demand level for low demands, but decreases in higher demand levels. 


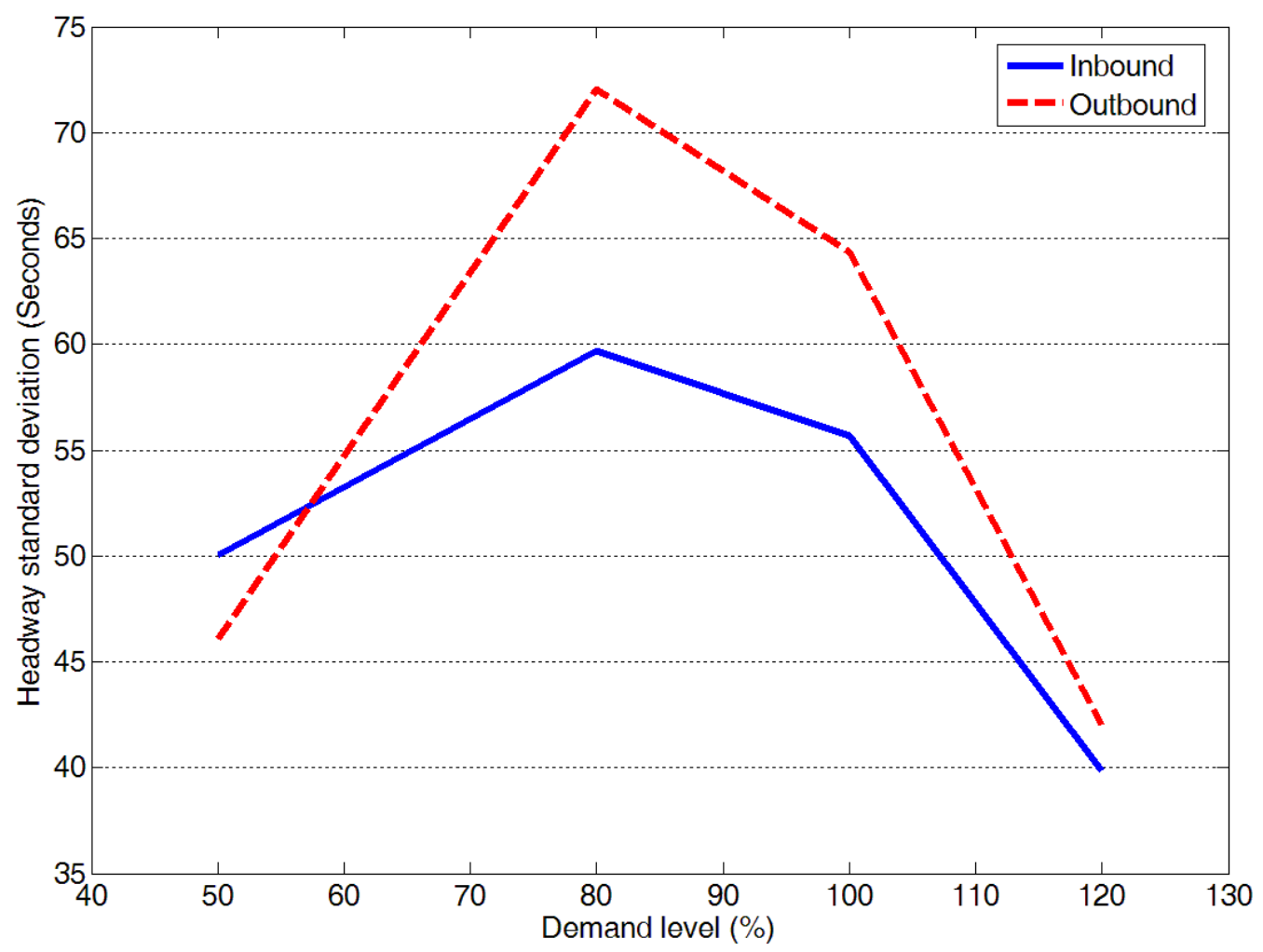

Figure 10: Standard deviation of headways as function of the demand level (inbound route)

Another important measure of service reliability is on-time performance. On-time performance was measured for all trips and all stops. The relatively low on-time performance, except for the high-demand scenarios, is because of early arrivals. The relative high share of early arrivals from the total number of buses that did not arrive on-time, calls for the implementation of schedule-based holding. The last system-level measure in Table 2 is the average number of passengers per stop that are unable to board the bus because it is overcrowded. As expected, this statistic increases with the level of passenger demand.

The objective of fleet assignment procedures is to generate trip chains with the minimal number of vehicles required to fulfill the schedule. This objective is better served by shorter 
layover and recovery times. However, the operator has to balance between the economic criteria and the level of service criteria, since shorter layover and recovery times will result in late departures, missed trips and poor on-time performance. Table 4 summarizes the results of a sensitivity analysis showing the impact on performance of different recovery time policies. (e.g. a policy of the $70^{\text {th }}$ percentile implies that trips will be chained according to the $70^{\text {th }}$ percentile of their running time distribution). The results demonstrate that as the recovery times decrease, the number of late departures increases. For example, a reduction of $20 \%$ in the number of buses used (from 15 to 12 ) results in $14 \%$ decrease in the on-time performance, $51 \%$ increase in the average schedule deviation and $162 \%$ increase in late departures from the origin terminal. The transit simulation supports the evaluation of this trade-off in order to identify optimal strategies through associating cost with bus vehicle allocation and schedule adherence

Table 4: Sensitivity analysis of recovery time policy

\begin{tabular}{|c|c|c|c|c|}
\hline $\begin{array}{c}\text { Recovery time (percentile } \\
\text { of run time) }\end{array}$ & Fleet & On-time & Schedule & Late \\
\hline $55 \%$ & 12 & 61.1 & 146 & 20.6 \\
\hline $70 \%$ & 14 & 67.3 & 134 & 9.5 \\
\hline $85 \%$ & 15 & 71.1 & 97 & 7.9 \\
\hline
\end{tabular}




\section{CONCLUSION}

This paper presents a transit simulation model based on the platform of an event-based mesoscopic traffic simulation model, Mezzo. The developed simulation represents schedules, trip chains, boarding and alighting processes, passengers left behind, dwell time, layover and recovery time and trip chaining. Furthermore, the model also captures the propagation of delays through the system and from trip to trip.

The capabilities of Mezzo as an evaluation tool of transit operations planning and control have been demonstrated with an application to a real-world high-demand line in the Tel Aviv metropolitan area. The case study shows results validating the performance of the simulation model and demonstrates the value of the implementation of bus operations and the kind of outputs that are generated by the simulation. Moreover, the model reproduces important phenomena such as propagation of headway variability along the route and bus bunching validated with real-world data. The simulation model has yet to be tested on realistic systemwide networks. Further developments of Mezzo focus on modeling of various control strategies, such as holding and expressing, with application to real-time control and on the enhancement of the representation of passenger demand and behavior.

\section{REFERENCES}

Abkowitz, M. and J. Tozzi (1987). Research contributions to managing transit service reliability. Advanced Transportation J., 21, 47-65.

Algers, S., E. Bernauer, M. Boero, L. Breheret, M. Dougherty , K. Fox and J.F. Gabard (1997). A review of micro-simulation models. Institute for Transport Studies, University of Leeds. 
Boxill, S.A. and L. Yu (2000). An evaluation of traffic simulation models for supporting ITS development. Center for Transportation training and Research, Texas Southern University.

Bowman L.A. and Turnquist M.A. (1981). Service frequency, schedule reliability and passenger wait times at transit stops. Transportation Research Part A, 15 (6), 465-471.

Burghout, W. (2004). Hybrid microscopic-mesoscopic traffic simulation. Doctoral Dissertation, Royal Institute of Technology, Stockholm, Sweden. http://kth.divaportal.org/smash/record.jsf?pid=diva2:14700

W. Burghout, H. Koutsopoulos, I. Andréasson (2005) Hybrid mesoscopic-microscopic traffic simulation, Transportation Research Record 1934, 218-225.

W. Burghout, H. N. Koutsopoulos and I. Andreasson (2006), A discrete-event mesoscopic traffic simulation model for hybrid traffic simulation, Proc. IEEE Intelligent Transportation Systems Conference, Toronto.

W. Burghout and J. Wahlstedt, (2007) Hybrid traffic simulation with adaptive signal control, Transportation Research Record 1999, 191-197.

Casey R.F., Labell L.N., Moniz L. et al. (2000). Advanced Public Transportation Systems The state of the art: Update 2000. U.S. Department of Transportation, Federal Transit Administration (FTA), Washington DC.

Ceder, A. (2007). Public transit planning and operation: Theory, modelling and practice, Elsevier.

Chang, J., J. Collura, F. Dion and H. Rakha (2003). Evaluation of service reliability impacts of traffic signal priority strategies for bus transit. Transportation Research Record, 1841, 23-31.

Dessouky, M., R. Hall, L. Zhang and A. Singh (2003). Real-Time control of buses for schedule coordination at a terminal. Transportation Research Part A, 37, 145-164. 
Ding, Y., S. Chien and A. Zayas (2001). Simulating bus operations with enhanced corridor simulator. Transportation Research Record, 1731, 104-111.

Fernandez, R., C.E. Corts and V. Burgos (2007). Modelling passengers, buses and stops in traffic microsimulators: The MISTRANSIT approach on the PARAMICS platform. Preprints of the Transportation Research Board $86^{\text {th }}$ annual meeting, Washington DC.

Fu, L. and X. Yang (2002). Design and implementation of bus-holding control strategies with real-time information. Transportation Research Record, 1791, 6-12.

Khasnabis, S., R.R. Karanti and R.K. Rudraraju (1996). NETSIM-based approach to evaluation of bus preemption strategies. Transportation Research Record, 1554, 8089.

Kittelson and Associates, Texas Transport Institute, Transport Consulting Limited (2003). Transit Capacity and Quality of Service Manual, 2nd Edition. Transit Cooperative Research Program. TCRP Report 100, Washington DC.

Lee, J., A. Shalaby, J. Greenough, M. Bowie and S. Hung (2005). Advanced transit signal priority control using on-line microsimulation-based transit prediction model. Preprints of the Transportation Research Board $84^{\text {th }}$ annual meeting, Washington, DC.

Lin, T. and N.H.M Wilson (1992). Dwell time relationships for light rail systems. Transportation Research Record, 1361, 287-295.

Liu, R., S. Clark, F. Montgomery and D. Watling (1999). Microscopic modelling of traffic management measures for guided bus operation. Proceedings of $8^{\text {th }}$ World Conference on Transport Research, Antwerp, Belgium.

Liu, R., D. Van Vliet and D. Watling (2006). Microsimulation models incorporating both demand and supply dynamics. Transportation Research Part A , 40, 125-150.

Liu G. and Wirasinghe S.C. (2001). A simulation model of reliable schedule design for a fixed transit route. Journal of Advanced Transportation, 35 (2), 145-174. 
Morgan, D.J. (2002). A microscopic simulation laboratory for advanced public transport system evaluation. Master Thesis, Massachusetts Institute of Technology.

Nakanishi Y.J. (1997). Bus performance indicators - On-time performance and service regularity. Transportation Research Record 1571, 3-13.

Silva, P.C.M. (2001). Modelling interactions between bus operations and traffic flow. Doctoral Dissertation, University College London.

Taylor, M.A.P. (1982). Travel time variability: The case of two public modes. Transportation Science, 16, 507-521

TCRP (2003). Transit capacity and quality of service manual (TCQSM) $2^{\text {nd }}$ edition. Transportation Research Board, TCRP Report 100, Washington, DC.

Werf, J.V. (2004). SmartBRT: A tool for simulating, visualizing and evaluating bus rapid transit systems. California PATH Research Report, Institute of Transport Studies, University of California, Berkeley. 